\title{
Erratum to: Elimination of extremal index zeroes from generic paths of closed 1-forms
}

\author{
Carlos Moraga Ferrándiz
}

Published online: 8 October 2014

(C) Springer-Verlag Berlin Heidelberg 2014

\section{Erratum to: Math. Z. DOI 10.1007/s00209-014-1332-4}

In the original publication, the theorem number 4 was incorrectly cited as theorem 1 , in page 3 , below the heading "Theorem 4". The correct text should read as follows.

The notion of simple equivalence can be found on [14]. The homology of the complex $C_{*}\left(\alpha, \partial_{*}^{\xi, B}\right)$, which we denote by $H_{*}(M, u)$, only depends on the class $u$ and it is called the Novikov homology of the class $u$. The historical reason is that the first theorem with the flavour of theorem 4 was given by Novikov in his foundational paper [18], which first gave Morse-type inequalities for $\mathbb{S}^{1}$-valued functions $f: M \rightarrow \mathbb{S}^{1}$. These inequalities are related to a homology theory of an abelian cover associated with $f$. Later, Sikorav proved in [20, Ch. IV] that the homology defined by Novikov is indeed a homology with local coefficients and extended it to non-abelian covers.

Latour also proved that $H_{*}(M, u)$ coincides with the version of Novikov homology on the universal cover defined in Sikorav's thesis.

Further versions of theorem 4 can be found on [19, Ch. 14, Th. 2.2 and Th. 2.4] and on [4, Th. 3.1].

The online version of the original article can be found under doi:10.1007/s00209-014-1332-4.

C. Moraga Ferrándiz $(\varangle)$

Graduate School of Mathematical Sciences, The University of Tokyo, 3-8-1 Komaba Meguro-ku,

Tokyo 153-8914, Japan

e-mail: carlos@ms.u-tokyo.ac.jp 